\title{
L'ascension des conservateurs et le traitement de la délinquance aux États-Unis : le cas du Violent Crime Control and Law Enforcement Act (1994)
}

Diane Bénédic-Meyer

\section{(2) OpenEdition \\ Journals}

Electronic version

URL: http://journals.openedition.org/chs/1674

DOI: $10.4000 /$ chs. 1674

ISSN: 1663-4837

Publisher

Librairie Droz

\section{Printed version}

Date of publication: 1 December 2016

ISBN: 978-2-600-01953-8

ISSN: $1422-0857$

\section{Electronic reference}

Diane Bénédic-Meyer, «L'ascension des conservateurs et le traitement de la délinquance aux ÉtatsUnis : le cas du Violent Crime Control and Law Enforcement Act (1994)», Crime, Histoire \& Sociétés / Crime, History \& Societies [Online], Vol. 20, $n^{\circ} 2$ | 2016, Online since 01 December 2018, connection on 02 May 2019. URL : http://journals.openedition.org/chs/1674 ; DOI : 10.4000/chs.1674

This text was automatically generated on 2 May 2019.

(c) Droz 


\section{L'ascension des conservateurs et le traitement de la délinquance aux États-Unis : le cas du Violent Crime Control and Law Enforcement Act (1994)}

Diane Bénédic-Meyer

\section{Introduction}

1 Lors de la convention nationale du parti républicain de 1992, l'ultraconservateur Pat Buchanan, ancien conseiller des présidents Nixon, Ford et Reagan, affirme l'existence d'une guerre culturelle (culture war) aux États-Unis. Buchanan termine son discours en incitant les plus courageux à faire entendre leur voix et à dire non au désordre moral et social. Il illustre son propos en évoquant le comportement exemplaire de deux jeunes gens durant les émeutes sanglantes de Los Angeles de la même année ${ }^{1}$.

Mes amis, cette élection porte sur quelque chose de bien plus important que sur simplement qui remporte quoi. Elle porte sur qui nous sommes. Elle porte sur nos croyances. Elle porte sur ce que nous défendons en tant qu'Américains. Une guerre religieuse touche notre pays pour l'âme de l'Amérique. C'est une guerre culturelle, aussi déterminante pour le futur de notre nation que la Guerre Froide l'était en son temps. (...) Mes amis, au cours de ces 25 dernières merveilleuses semaines, les jours les plus tristes furent ceux des émeutes sanglantes de Los Angeles, les pires de notre histoire. Mais même de cette tragédie affreuse peut surgir un message d'espoir. (...) Il y avait par exemple ces garçons de 19 ans prêts à mettre leur vie en péril pour empêcher une bande de malfrats de malmener des personnes âgées alors qu'ils ne les connaissaient même pas. Et comme ils reprenaient les villes de LA, maison après maison, nous devons de la même manière reprendre le contrôle de nos villes, de notre culture, et de notre pays ${ }^{2}$. 
2 La guerre culturelle que décrit Buchanan est un combat pour définir l'identité nationale américaine. Le concept de guerre culturelle peut être défini comme un conflit de valeurs et d'idées qui donne lieu à une polarisation sociale et politique. Son nom vient de l'allemand Kulturkampf qui désignait la confrontation entre le chancelier réformateur Bismarck et l'Église catholique allemande des années 1870 . Ce concept a fait l'objet de nombreuses études depuis la publication de l'ouvrage Culture Wars: The Struggle to Define America du sociologue James Hunter en 1991. Hunter fait part du conflit portant sur le futur de la nation américaine et qui oppose deux cadres de pensée. Selon lui, Dieu et la loi morale sont au centre du cadre de la pensée «orthodoxe " tandis que la raison et l'adaptation à la société contemporaine sont les principes qui guident le cadre de la pensée "progressiste ». Buchanan estime que les conservateurs doivent contrer l'action destructrice de l'aile gauche américaine. Il drape son discours politique dans un message populiste où l'Américain travailleur des classes moyennes est devenu la proie des élites dispendieuses et laxistes de gauche.

3 Le discours de Buchanan constitue une bonne entrée en matière pour étudier le rapport des conservateurs américains à la délinquance et les effets de cette relation sur le traitement de la délinquance dans la société américaine. Avant d'entrer plus en avant dans notre sujet, un effort de définition s'impose. Le conservatisme moderne américain s'inscrit par contrepoint au progressisme. Il est généré, d'une part, par l'opposition aux contraintes du marché et une conception individualisée de la liberté politique et, dans le même temps, nourri par sa dévotion à l'ordre social et sa foi religieuse. Le mouvement conservateur est de nature protéiforme car il s'est construit sous l'action d'intellectuels, de citoyens sans mandat et de professionnels de la politique. Il est à l'origine d'une droitisation quasi ininterrompue du parti républicain. Par opposition, le bloc progressiste, aussi appelé « liberal $»^{3}$, entend assurer la sécurité économique et sociale et la protection des droits de l'individu en ayant recours à une plus grande intervention de l'État. Contrairement aux conservateurs, les membres du bloc progressiste pensent qu'il est nécessaire de discipliner le marché et non l'individu.

4 Le thème de la loi et de l'ordre fait partie de la rhétorique politique du mouvement conservateur depuis ses débuts dans les années 1950. Le traitement de la délinquance par les conservateurs a suivi une évolution binaire. Il a d'abord été tributaire du «penal welfarism ", conception selon laquelle la délinquance est à penser comme une forme de carence interne ou externe à l'individu (le tenant du conservatisme étant plus enclin à croire en la carence interne). Puis dès la fin des années 1960, les conservateurs s'emparent plus franchement $\mathrm{du}$ thème de la loi et de l'ordre et demandent un renforcement des sanctions.

5 La modernité tardive de la décennie suivante et son cortège de transformations économiques (liberté de marché), sociales et culturelles entraînent une augmentation de la délinquance et par conséquent une modification de son traitement. Désormais, le crime est perçu comme un phénomène inévitable qu'il appartient de réguler ${ }^{4}$. De nouveaux modèles de justice pénale et de contrôle de la délinquance s'installent. Ils sont les composantes d'une «?culture du contrôle ?» identifiée comme telle par le sociologue et politologue David Garland ${ }^{5}$. La gestion du risque et la sécurité des personnes priment désormais sur la réinsertion et la gestion sociale. Le ralliement de la nouvelle gauche (les Nouveaux démocrates) à la politique sécuritaire dans les années 1990 confirme le bienfondé et la pérennisation de la grille de lecture de la nouvelle pénologie aux États-Unis. 
En effet, les Nouveaux démocrates se construisent en réaction au danger électoral que représente le mouvement conservateur.

6 Notre étude s'inscrit dans cette seconde phase du traitement de la délinquance. Nous proposons d'étudier le cas du Violent Crime Control and Law Enforcement Act (VCCLEA) de 1994. Il s'agit de la loi de lutte contre la criminalité la plus ambitieuse de la seconde moitié du XXe siècle tant en termes de budget que de contenu. Le VCCLEA prévoit notamment le renforcement des forces de l'ordre et du système carcéral, le maintien des dépenses pour combattre les réseaux liés à la drogue mais aussi la mise en place d'une police communautaire, la protection des femmes victimes de violence et l'interdiction des fusils d'assaut.

7 Nous exposerons d'abord comment les conservateurs se sont progressivement emparés du discours politique sur la criminalité depuis 1964 jusqu'à présenter le parti républicain comme l'unique recours face à l'insécurité. Nous verrons ensuite pourquoi le VCCLEA est symptomatique d'une droitisation de la vie politique américaine en exposant ses racines conservatrices et progressistes. La troisième partie traitera de la bataille législative acharnée dont le VCCLEA fit l'objet. L'accent sera mis sur l'obstructionnisme dont firent preuve les républicains $\mathrm{du} 103^{\mathrm{e}}$ Congrès et qui aboutit à la révolution conservatrice parlementaire de 1994. La dernière partie de cette étude portera sur la réévaluation du VCCLEA plus de vingt ans après qu'il ait été voté. Que nous apprend cette réévaluation sur l'état du traitement de la délinquance à l'aube de l'élection présidentielle de 2016 ? Annoncerait-elle l'avènement d'une nouvelle et troisième phase du traitement de la lutte contre la délinquance aux États-Unis?

\section{Le discours politique sur la criminalité et l'insécurité : un sujet républicain ? (1964-1988)}

8 La stratégie de conquête électorale du mouvement conservateur passe par la préemption de certains thèmes de campagne dont la lutte contre la criminalité fait partie. Pour mieux comprendre l'évolution du discours des partis républicains et démocrates sur la question de l'insécurité, nous aurons recours aux travaux de John Petrocik et Theodore Sasson, deux spécialistes américains reconnus en sciences politiques et en sociologie.

9 Le débat autour de l'appartenance d'un sujet à un parti politique a fait l'objet de nombreuses études au cours des années 1980 et $1990^{6}$. La théorie de l'appartenance d'un sujet (issue ownership) telle qu'elle a été énoncée par John Petrocik, affirme que les partis politiques peuvent se forger une réputation de compétence dans un domaine particulier. Petrocik définit l'appartenance d'un sujet comme «la capacité à résoudre un problème qui importe aux électeurs ». Le parti politique a bâti une "réputation » en construisant une "histoire basée sur l'attention, l'initiative, et l'innovation pour résoudre ces problèmes ${ }^{7}$. En d'autres termes, un parti peut chercher à devenir si intimement associé à un sujet que l'électeur aura tendance à prendre pour acquise la compétence de ce dernier en la matière. Petrocik distingue les sujets qui appartiennent au Parti démocrate comme l'aide sociale, les droits des femmes, des minorités et des populations défavorisées de ceux qui relèvent de la sphère des républicains comme la réduction de l'intervention de l'État fédéral, l'ordre civil et social, ainsi que les valeurs morales ${ }^{8}$. Une seconde vague d'études au début des années 2000 a confirmé le bien-fondé de la théorie de Petrocik pour

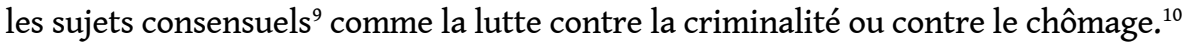


10 À présent que la rivalité pour l'appartenance du sujet de la lutte contre la criminalité entre républicains et démocrates a été posée, il convient de se pencher sur les cadres de pensée que chaque parti utilise le plus fréquemment. En 1995, Theodore Sasson a identifié cinq cadres de pensée : le système défectueux (faulty system), la rupture sociale (social breakdown), les opportunités bloquées (blocked opportunities), la violence des médias (media violence) et le système raciste (racist system). Les cadres du système défectueux, de la violence des médias et du système raciste servent couramment l'idéologie ${ }^{11}$ des conservateurs tandis que les démocrates ont traditionnellement recours à ceux de la rupture sociale et des opportunités bloquées.

11 Dès 1960, Barry Goldwater, sénateur républicain encore méconnu, appelle les conservateurs à conquérir le Parti républicain : «Grandissons, les conservateurs, si nous voulons reprendre ce parti, et je pense que nous le pourrons un jour. Mettons-nous au travail ». En 1964, la mise à l'écart de Nelson Rockfeller, candidat de la frange libérale plus modérée des républicains, marque le début de la mainmise du mouvement conservateur sur le Grand old Party (GOP). L'élection présidentielle qui l'oppose au président Lyndon B. Johnson, est l'occasion pour Goldwater de faire de la lutte contre le crime un thème fédérateur. S'il veut réussir, il lui faut exposer le caractère irréconciliable des cadres de pensée conservateurs et progressistes. En vérité, les chances de victoire de Goldwater sont minces face à la popularité immense de Johnson. L'institut de sondage Gallup indique que $74,2 \%$ des Américains approuvent la manière dont Johnson remplit la fonction présidentielle de novembre 1963 à janvier 1965. Les circonstances exceptionnelles de son arrivée au pouvoir après l'assassinat de John F. Kennedy expliquent en partie ce chiffre. La stratégie de Goldwater consiste à affaiblir les démocrates du Sud et à fédérer les différentes composantes du conservatisme. Le discours de Goldwater devait pouvoir plaire à la fois aux conservateurs sociaux ou culturels (social conservatives, cultural conservatives) et aux tenants d'un conservatisme fiscal (fiscal conservatives). Certes, ces deux groupes sont sensibles aux droits des États mais les conservateurs culturels entendent conserver l'immuabilité de la société en protégeant la norme et les valeurs traditionnelles tandis que les conservateurs fiscaux défendent la rigueur budgétaire avant tout.

12 Le thème de la lutte contre la criminalité présente un potentiel populiste qui n'a pas échappé à Goldwater. L'argumentation politique qu'il développe en 1964 marque le début d'une approche nouvelle des élus conservateurs, lesquels vont s'enhardir à demander un durcissement de l'intervention de l'État. Comme le rappelle Sophie Body-Gendrot, spécialiste française des questions urbaines, la sanction du crime rassure la majorité morale en jouant sur la peur de l'Autre ${ }^{12}$. Goldwater évoque le thème du retour à la loi et à l'ordre dans son discours d'investiture : «Ce soir il y a de la violence dans nos rues, de la corruption dans notre haute administration, une perte de sens chez nos jeunes, de l'angoisse chez nos aînés (...) » $»^{13}$. Il joue sur la méfiance des républicains à l'endroit de l'État fédéral et il adopte une stratégie de l'attaque ${ }^{14}$. Enfin, Goldwater espère s'attirer les bonnes grâces des électeurs du Sud en associant le mouvement des droits civiques à l'image des transgresseurs de loi. Il introduit par ce biais le facteur racial qui trouve un écho dans l'imagerie sudiste du criminel noir menaçant les familles blanches et les femmes en particulier.

De son côté, Lyndon Johnson assimile la problématique de la criminalité à une question d'ordre social. Il conçoit la criminalité comme la conséquence de l'inégalité et de la discrimination telles qu'elles se manifestent sur le lieu de travail, dans les écoles et dans 
les milieux défavorisés. À ses yeux, le programme de la guerre contre la pauvreté a entre autres visées de faire baisser les chiffres de la criminalité. Malgré la victoire écrasante de Johnson qui remporte 44 états et $61 \%$ du vote populaire, la bataille électorale pour la préemption du thème de la lutte contre la criminalité a mis à jour les dissensions géographiques et idéologiques au sein du Parti démocrate. Johnson en paye le prix. Il n'a d'autre choix que de négocier avec les démocrates du Sud pour faire voter une nouvelle loi anti criminalité, l'Omnibus Crime Control and Safe Streets Action de 1968. Or les positions des démocrates du Sud sont très proches de celles des républicains : tous deux souhaitent une répression plus stricte et notamment l'allongement des peines carcérales.

De fait, l'ascension des conservateurs va s'appuyer de plus en plus fortement sur la région du Sud. Du statut de bastion démocrate, le solid South ${ }^{15}$ devient à partir des années 1970 une terre de compétition électorale. Certes, la politique de Johnson en faveur de la défense des droits civiques a ouvert une brèche importante où les républicains auront tôt fait de s'engouffrer, mais ce sont aussi les transformations économiques et sociales $\mathrm{du}$ Sud qui sont à l'origine du réalignement d'une partie de son électorat en faveur du Parti républicain ${ }^{16}$. L'avènement d'une classe moyenne blanche $\mathrm{du}$ Sud réceptive à l'argumentaire du moins d'État, contribue grandement à l'avancée électorale des conservateurs. Ces derniers s'appuient sur l'accroissement du poids démographique ${ }^{17}$ et donc électoral du Sud pour renforcer le succès des candidats républicains aux élections présidentielles et législatives ${ }^{18}$. L'effort de conquête électorale des conservateurs contribue certainement à la progression, puis au maintien, d'une opinion publique qui se déclare davantage conservatrice que liberal (de gauche) depuis les années 1980. En 2014, soit près d'un demi-siècle après la signature du Civil Rights Act (1964) par Johnson, $38 \%$ des Américains se considéraient comme conservateurs ${ }^{19}$.

15 Après la tentative avortée de Goldwater, le discours sécuritaire des républicains se poursuit sous les présidences Nixon et Reagan. Aux élections présidentielles de 1968, le candidat Nixon traite du thème du crime non organisé. Il marque là un tournant puisque contrairement à la campagne de Robert Kennedy qui avait fait de la lutte contre le crime organisé son cheval de bataille en 1963, Richard Nixon s'attaque à la petite délinquance urbaine. On notera l'habileté de la manoeuvre puisque l'exécutif n'a que peu de prise sur la délinquance urbaine qui est du ressort des États et des collectivités locales. En 1970, Nixon use à nouveau du discours sécuritaire à des fins électorales. Il dénonce le laxisme de ses prédécesseurs démocrates et la fausseté de leur discours. Sa campagne présidentielle de 1968 est l'occasion de mettre en pratique une stratégie électorale visant à rallier l'électorat blanc conservateur du Sud (southern strategy). Tout comme Barry Goldwater, Nixon a recours à des thèmes porteurs comme le droit des États et la question sécuritaire. Signe des temps, Nixon ajoute à ce message la résistance à l'intégration raciale. Toutefois, la campagne de 1968 marque une étape nouvelle dans l'instrumentalisation de la question sécuritaire puisqu'elle s'accompagne d'une mise en cause de la Cour suprême. Nixon remet en question la décision de la Cour de nationaliser les procédures criminelles, et notamment l'arrêt Miranda v. Arizona de 1966, lequel oblige la police à informer un suspect de ses droits. La démission de Nixon consécutive au scandale du Watergate de 1972 marque une pause dans la politisation de la lutte contre la criminalité et la délinquance dans les campagnes présidentielles. Les Américains sont davantage préoccupés par la stagflation qui touche le pays. De plus, la lutte contre la criminalité est perçue comme étant la chasse gardée des républicains, y compris par les démocrates eux-mêmes qui évitent soigneusement d'aborder la question. 
16 Il faut attendre la campagne présidentielle de Ronald Reagan en 1980 pour que le sujet réapparaisse sur le devant de la scène nationale. Reagan avait déjà utilisé ce thème avec succès lors de sa campagne pour le siège de gouverneur de Californie. Selon lui, la criminalité relève de la responsabilité individuelle comme il le rappelle avec force à la convention républicaine de 1968 : « Nous devons rejeter l'idée que chaque fois que la loi a été violée, la société est coupable plutôt que le contrevenant. Il est temps de restaurer le précepte américain selon lequel chaque individu doit répondre de ses actes $»^{20}$. Une fois président, Reagan dirige ses efforts dans le domaine de la lutte contre la drogue, allant jusqu'à utiliser l'image de son épouse Nancy dans sa campagne de communication ${ }^{21}$. Il fait voter deux lois anti-drogue en 1986 et 1988 qui durcissent la répression envers les trafiquants. Certes, des programmes de prévention existent mais ils sont dérisoires face aux dépenses qui sont allouées aux forces de l'ordre. Les chiffres parlent d'eux-mêmes : en 1985, les forces de l'ordre bénéficient de $78 \%$ du budget global dans la lutte contre la drogue $^{22}$. Cette politique répressive s'inscrit dans la conception néolibérale du traitement de la délinquance, qui associe une croyance dans les idéaux du libre marché (le laissez faire) à une demande accrue d'intervention de l'État dans la sphère pénale. Ce paradoxe apparent est en partie élucidé si l'on pense qu'un État suffisamment fort est le seul à même de garantir le bon fonctionnement du système économique libéral. Dans la pensée des conservateurs américains, le crime est compris comme une violation des règles du marché23. Par ailleurs, Reagan poursuit l'avancée des conservateurs au sein de la Cour suprême par une politique de nominations de juges d'obédience conservatrice.

La campagne présidentielle de 1988 fait encore monter d'un cran l'intensité des attaques contre les démocrates sur la question sécuritaire. Sophie Body-Gendrot utilise l'image du «?triangle de fer ?» pour expliquer la stratégie républicaine qui vise à convaincre le citoyen du bien-fondé de la répression. Ce triangle combine les messages des hommes politiques, des médias et des groupes de pression ${ }^{24}$. Les républicains font preuve d'une grande efficacité en faisant de Dukakis, le candidat démocrate de 1988, l'illustration parfaite du laxisme de la gauche sur la question sécuritaire. Les républicains profitent en particulier de la mauvaise prestation de Dukakis au second débat télévisé de l'élection présidentielle pour faire de la peine de mort une question décisive (litmus test). Dukakis n'a pourtant pas à rougir des bons résultats de son État du Massachusetts car le taux de criminalité y est inférieur à la moyenne nationale ${ }^{25}$. Dukakis reste fidèle à son positionnement contre la peine de mort pendant le débat. À la question «si votre épouse Kitty était victime d'un viol puis assassinée, demanderiez-vous la peine de mort pour l'assassin?», le gouverneur répond qu'il ne croit pas en la peine de mort. Malgré l'étrangeté de la question, c'est bel et bien la réponse dénuée d'émotion de Dukakis qui porte un coup d'arrêt fatal à sa campagne.

18 La campagne des républicains est aussi marquée par l'utilisation agressive de l'outil médiatique. S'il n'en est pas à l'origine, le directeur de campagne de George H. Bush, Lee Atwater, voit d'un bon oeil la diffusion du spot «Willie Horton » par le comité d'action politique pour la sécurité nationale (National Security Political Action Committee). Il considère - à juste titre - l'électorat conservateur du Sud comme un élément clé de la victoire. Le spot associe l'image de Dukakis à l'affaire du criminel récidiviste noir Willie Horton. En 1986, Horton profite d'une permission de l'État du Massachusetts pour s'échapper. Dix mois plus tard, il se rend dans le Maryland où il commet un nouveau forfait et torture un jeune couple à son domicile. Horton sera finalement condamné à la prison à vie. Sur la dernière image du spot figurent les mots suivants : «Weekend prison 
passes: Dukakis on crime $\aleph^{26}$. Le traitement de l'affaire Horton par les républicains contient trois messages : il stigmatise l'échec du traitement de la gauche dans la lutte contre la criminalité, il nourrit les peurs de l'opinion publique, et il donne un caractère racial et social au crime en lui prêtant les traits d'un homme noir issu d'un milieu défavorisé. Le spot eut un tel impact que son souvenir a perduré dans les esprits longtemps après sa diffusion. La facilité avec laquelle les républicains ont pu jouer avec le thème de la lutte contre la criminalité dans la campagne de George $\mathrm{H}$. Bush démontre que les républicains se sont bel et bien appropriés cette thématique à l'orée des années 1990.

\section{Les racines conservatrices et progressistes du Violent Crime Control and Law Enforcement Act (1982-1993)}

Les années 1990 sont l'occasion d'un renouveau du traitement de la criminalité par les démocrates les plus modérés. Au début des années 1990, le taux de criminalité aux ÉtatsUnis est près de quatre fois supérieur à la moyenne de l'Europe de l'Ouest. Le Bureau of Justice Statistics recense quatorze millions de crimes en 1991. Il met aussi en lumière une accélération de la progression du crime de l'ordre de $23 \%$ entre 1988 et 1991. Depuis la défaite de George McGovern aux élections présidentielles de 1972, les démocrates au progressisme le plus modéré cherchent à faire entendre leur voix avec plus de force. Ils contestent les dérives du déplacement vers la gauche du progressisme américain lequel favorise les groupes d'intérêts au détriment des élus. L'élection présidentielle de Jimmy Carter en 1976 entraîne dans son sillage l'arrivée d'une nouvelle génération d'élus au progressisme plus modéré parmi les gouverneurs et les parlementaires du Congrès. Certains entendent se saisir à nouveau du thème de la lutte contre la criminalité car la mainmise républicaine sur la question est devenue difficilement supportable. Les élus démocrates blancs du Sud se sentent menacés par le réalignement de l'électorat que promettent les républicains alors que le mouvement conservateur gagne du terrain.

Nous nous arrêterons sur le parcours de deux réformateurs démocrates influents: Joe Biden, sénateur du Delaware (et futur vice-président au sein de l'administration Obama) et Bill Clinton, gouverneur de l'Arkansas qui deviendra le $42^{\mathrm{e}}$ président des États-Unis. L'action combinée de ces deux hommes rendra possible le vote du Violent Crime Control and Law Enforcement Act (VCCLEA) de 1994. Notre étude se limitera à la question sécuritaire. Celle-ci entre dans le cadre du déplacement vers la droite du centre de gravité idéologique du Parti démocrate auquel clinton et Biden ont contribué : le premier avec la réforme de l'aide sociale de 1996 et le second alors qu'il présidait le comité sénatorial des relations étrangères sous l'administration de George W. Bush (2001-2003, 2007-2009).

21 Le VCCLEA est tributaire des travaux de George L. Kelling et de James Q. Wilson (1931-2012), spécialistes américains des questions urbaines qui ont inspiré à la fois les conservateurs des années 1980 et les démocrates modérés. En mars 1982, Kelling a publié en collaboration avec Wilson un article éponyme qui présente la théorie des «vitres cassées " dans The Atlantic Monthly. Ils y démontrent que l'état de délabrement d'un endroit public contribue au sentiment d'insécurité et à attirer l'implantation de criminels. L'article connaît un certain succès d'autant plus que Kelling officie en qualité de conseiller pour l'administration Reagan. Cette théorie donne lieu à des expériences de réhabilitation des lieux publics dans le New Jersey au milieu des années 1980. George Kelling rejoindra les rangs du think tank conservateur The Manhattan Institute et 
s'illustrera auprès des républicains en assistant le maire Rudolph Giuliani dans la sécurisation du métro de New York en 1995 (avec une présence policière accrue et l'application de la tolérance zéro).

Du côté démocrate, le Democratic Leadership Council, un réseau d'élus démocrates modérés, s'efforce depuis sa création en 1985 de moderniser le progressisme du parti de l'âne en le déportant plus à droite. Bill Clinton et Joe Biden en rejoignent les rangs à la fin des années 1980. Bill Clinton en devient le porte-parole en 1991. Au cours de sa campagne présidentielle de 1992, il adopte la posture de Nouveau démocrate initiée par le Democratic Leadership Council (DLC). Biden se montre plus discret quant à son attachement au DLC durant ses années sénatoriales ${ }^{27}$. En 1990, le tout jeune think tank du DLC, le Progressive Policy Institute, propose la création de Police Corps, un programme de service civil au sein des forces de police. Le programme repose sur le principe de la police de proximité ( community police $\left.{ }^{28}\right)$. Ce dernier peut être défini comme une philosophie de pratique de la police, placée sous le signe de la coopération entre les officiers de police et la population civile du quartier concerné, pouvant contribuer à résoudre des problèmes locaux liés au crime, à la peur du crime, à la qualité de vie, et aux conditions de vie du voisinage ${ }^{29}$. Aux États-Unis, le concept de la police de proximité est l'héritier de deux traditions, celle des community watchmen, des patrouilles de civils qui arpentent le territoire de la communauté pour dissuader les criminels éventuels depuis l'apparition des premières colonies britanniques, et celle des vigilante, des patrouilles de citoyens qui jouaient le rôle d'agents de l'ordre (policiers et juges confondus) dans les nouveaux territoires de l'expansion américaine du XIX ${ }^{\mathrm{e}}$ siècle.

Pour être plus précis, les Nouveaux démocrates reprennent le discours des penseurs communautaires. Les communautaristes se reconnaissent dans les idées républicaines du début de la République américaine qui ont à la fois pour aspiration l'avancement du bien commun, la liberté et la vertu civique. Selon eux, les sociétés humaines doivent être comprises comme des communautés qui créent, partagent, divisent et échangent un éventail de «bien sociaux » (social goods). Tout État serait par principe un État providence et chaque société déciderait de la nature des biens sociaux en fonction des besoins de ses membres. Les communautaires posent la sécurité et le bien-être social comme les deux principaux biens sociaux de la société américaine contemporaine ${ }^{30}$. Face à la guerre culturelle nourrie par la rhétorique des conservateurs, ils ressentent avec acuité l'absence de consensus national quant à la définition de la notion de bien commun. Leur manifeste de 1995 permet de mieux appréhender leur pensée :

De manière plus profonde, nous étions troublés par le fait que de nombreux Américains se montrent plutôt réticents à accepter les responsabilités. Nous étions peinés de voir que de nombreux Américains étaient vivement désireux de réclamer haut et fort ce à quoi ils avaient droit, mais étaient lents à donner quelque chose en retour à la communauté et aux autres. Nous avons adopté le nom de 'communautaire' pour souligner le fait que le temps est venu de faire face à nos responsabilités vis-à-vis de nos communautés ${ }^{31}$.

La participation active du citoyen pour assurer le bien commun (ici la sécurité du quartier) que les Nouveaux démocrates plébiscitent s'inscrit donc pleinement dans le renouvellement de la communauté préconisé par les communautaires des années 1980 et 1990.

Proche du mouvement des Nouveaux démocrates, Joe Biden s'inquiète aussi de la polarisation qui touche le monde politique. Le sénateur du Delaware montre un intérêt constant pour la lutte contre la délinquance. Il occupe la présidence du comité judiciaire 
du Sénat depuis 1987. La hausse inquiétante du taux national de criminalité le conduit à s'intéresser à la lutte contre le trafic de drogue. En 1990, Biden présente un premier projet de loi contre le crime. Il demande à cette occasion l'augmentation des effectifs de police, l'interdiction des armes d'assaut et le durcissement des peines encourues par les trafiquants de drogue. Le projet de loi ne manque pas de susciter la désapprobation des républicains et du puissant lobby des armes de la National Rifle Association (NRA). Il n'y a rien d'étonnant à cela puisque la NRA est partie prenante dans l'ascension des conservateurs. Le lobby des armes s'oppose farouchement à la réglementation des armes depuis le début du débat moderne sur la question lorsque Lee H. Oswald (1939-1963) se servit d'une arme achetée via une publicité dans un journal contre le président Kennedy ${ }^{32}$ . Toutefois, c'est une coalition contre nature composée d'une forte proportion de républicains et de quelques démocrates parmi les plus à gauche qui parvient à bloquer au Sénat le projet de loi de Biden. Trois ans plus tard, dans son discours d'introduction du VCCLEA, l'intéressé mettra son échec sur le compte du climat partisan qui régnait au $101^{\mathrm{e}}$ Congrès :

Pendant trop longtemps, une majorité de ce corps a préféré le politique au pragmatique. Lors du dernier Congrès, nous avions l'occasion de voter pour une législation anti criminalité attendue qui avait reçu l'aval des démocrates et des républicains pour chacun de ses programmes. Au lieu de quoi nous sommes demeurés obnubilés par nos désaccords sur des détails qui ont allumé l'étincelle de la chaleur partisane dans des proportions exagérées par comparaison à l'importance de combattre le crime avec efficacité ${ }^{33}$.

Joe Biden ne s'avoue pas vaincu. Il multiplie les auditions au Sénat pour proposer un nouveau projet de loi de 1991 à 1993. Il prend alors la mesure des violences faites aux femmes ainsi que celle du vide législatif qui sévit dans le domaine. Biden souhaite par conséquent élever le crime contre les femmes au rang de crime haineux (hate crime) afin qu'il soit considéré comme une violation des droits civiques au même titre que les crimes raciaux. Joe Biden se heurte rapidement à William H. Rehnquist (1924-2005), le très conservateur président de la Cour Suprême ${ }^{34}$ et aux groupes religieux de droite. En revanche, Biden reçoit parmi les républicains le soutien de son confrère Orrin G. Hatch (né en 1934) avec qui il alterne la présidence du comité judiciaire au Sénat. Biden obtiendra finalement gain de cause en 1994 lorsqu'il recevra l'appui du président Clinton pour le vote du VCCLEA.

Bien que Joe Biden et Bill Clinton aient tous deux oeuvré pour faire aboutir le VCCLEA, leur réflexion sur la lutte contre le crime a pris des chemins différents. Bill clinton est issu d'un État du Sud et il est en tant que tel soumis à l'approbation d'un électorat plus conservateur que la ligne établie par l'establishment du Parti démocrate national. Au cours de son premier mandat de gouverneur, Clinton a appris à ses dépens qu'il lui fallait soigner une image sécuritaire forte. Confronté à la fuite d'immigrés illégaux cubains internés par l'administration fédérale à Fort Chaffee au nord-est de l'Arkansas, il fait son possible pour éviter les heurts avec la population locale. Clinton doit faire intervenir les forces de l'ordre de l'Arkansas face au laisser-aller des gardes fédéraux qui avaient laissé les Cubains quitter le fort et dénonce alors les déficiences de l'administration Carter. L'affaire des réfugiés cubains finit malgré tout par mettre en péril ses chances de réélection. Clinton ne retrouve son siège de gouverneur que deux ans plus tard. L'homme retient la leçon et adopte par la suite une posture de sévérité. Il modifie notamment sa ligne de conduite vis-à-vis des condamnés à mort. Alors qu'il avait commué la peine de 
soixante-dix condamnés à mort en celle de prison à vie pendant son premier mandat, Clinton ne réserve ce traitement qu'à sept personnes de 1984 à 1991.

En 1992, Clinton fait de la lutte contre la criminalité l'un des thèmes de sa campagne. Il s'agit là d'un choix courageux mais réfléchi. Il est hors de question pour lui de se faire taxer de laxisme comme son prédécesseur Michael Dukakis. La question sécuritaire demeure toutefois délicate et divise au sein des démocrates. Quelle qu'elle soit, la position de Clinton a toutes les chances de susciter les passions. Bill Clinton procède en deux étapes. Il suit tout d'abord les règles établies par les républicains dans le débat sur la question sécuritaire en se prononçant en faveur de la peine de mort. Il va jusqu'à arrêter sa campagne pour revenir en Arkansas et autoriser l'exécution de Rickey Rector dont il avait pourtant été établi qu'il ne possédait plus toutes ses capacités mentales. Puis il expose son propre traitement du thème de la lutte contre la criminalité. Son livre de campagne Mandate for Change établit un ensemble de mesures concrètes comme l'ajout d'un effectif de 100000 policiers, la création de centres de détention réservés aux jeunes ayant commis leur premier forfait, et des campagnes de prévention contre la drogue et d'accompagnement pour les toxicomanes. Clinton se prononce aussi en faveur d'une approche communautaire. Il encourage les initiatives qui incluent la participation du voisinage et la coopération entre la population et la police de proximité. Il souhaite la mise en application de Police Corps, un programme de service civil au sein des forces de police. Clinton envisage également des mesures qui relèvent d'un progressisme plus traditionnel comme l'interdiction de la vente des armes d'assaut et la régulation de leur circulation. Il se prononce enfin pour l'application de la loi Brady (Brady Handgun Violence Prevention Act), restée bloquée à la Chambre basse, qui préconise de vérifier les antécédents des acquéreurs d'armes.

Les républicains quant à eux se sentent floués car ils ont l'impression que Clinton a préempté une de leurs thématiques favorites. Clinton n'est bien entendu pas du même avis : «Quand je suis arrivé à Washington, je lisais des éditoriaux dans de grands journaux qui disaient que si vous vous souciez du déficit, de la criminalité et de l'aide sociale, vous voliez les sujets des républicains. Et j'ai dit, eh bien, attendez une minute. (...) Je pense que ce sont des sujets américains $(. . .)^{35}$ ", déclarera-t-il en 1997 devant une assemblée de gouverneurs démocrates. L'intention de Clinton est claire: il veut redéfinir le débat autour de la question sécuritaire en ses propres termes et en maitriser l'agenda ${ }^{36}$. Il fera de même pour la réforme de l'aide sociale de 1996 où il fera prévaloir le principe du workfare. Dans une société marquée par un virage néolibéral, il applique l'idée d'une contrepartie par le citoyen qui demande une aide de l'État, sous la forme d'une obligation de travail ou d'une formation professionnelle ${ }^{37}$. Pour ces deux thématiques sensibles de la sécurité et de l'aide sociale, Bill Clinton suit la philosophie politique des Nouveaux démocrates qui cherchent à trouver une approche qui sorte de la stricte polarisation gauche/droite et qui co-optent au besoin des idées chères au mouvement conservateur ${ }^{38}$.

\section{La bataille législative autour du VCCLEA (1993-94)}

Peu de temps après son accession à la présidence, Bill Clinton se trouve confronté à une très forte opposition pour faire voter le budget fédéral puis la réforme de santé qu'il a confiée à son épouse Hillary. Il lui semble donc opportun de reprendre les commandes de son agenda politique et de traiter d'un thème qu'il estime des plus porteurs, celui de la lutte contre la délinquance. Il s'attaque ce faisant à un bastion républicain. Le projet de 
loi du VCCLEA va se retrouver rapidement au centre d'une bataille législative qui, si elle est remportée par Clinton avec la signature du VCCLEA le 13 septembre 1994, servira finalement les intérêts de la nébuleuse conservatrice.

Le dossier de la lutte contre la criminalité est confié au Domestic Policy Council, un organe de l'exécutif. Ce dernier met en place une commission de réflexion qui soutient fermement l'action du sénateur Biden. Concrètement, le VCCLEA prévoit le recrutement de 100000 policiers en six ans, dont la moitié sera affectée à la police de proximité, ainsi que la création de camps pénitentiaires pour jeunes délinquants, et le durcissement du droit à l'habeas corpus pour les trafiquants de drogue. Il alloue un budget record de 9,7 milliards de dollars au système pénitentiaire. Le second volet est consacré aux violences faites aux femmes avec le Safe Street Violence Act qui accroît la sévérité des peines à l'encontre des récidivistes et le Safe Homes for Women Act qui prend mieux en compte les violences au sein du foyer.

Le vote pour le VCCLEA s'annonce difficile. Les républicains ne peuvent accepter ni la clause interdisant les fusils d'assaut ni le montant des dépenses liées à la prévention qu'ils estiment trop élevé. En vérité, des raisons plus profondes expliquent le refus des républicains. Afin de consolider leur avancée électorale, représentants et sénateurs républicains se sont engagés dans une stratégie d'obstruction parlementaire qu'ils ont déjà pratiquée lors du vote du budget. Ils avaient utilisé la flibuste dans le but avoué d'allonger les débats au Sénat ${ }^{39}$. Facteur aggravant, les républicains sont particulièrement remontés contre toute intervention touchant à la réglementation des armes à feu depuis que la loi Brady a été votée et a reçu la signature présidentielle le 30 novembre 1993.

Cependant, la faiblesse du VCCLEA est surtout due à l'étendue de son contenu. Les élus porteurs du VCCLEA, en accord avec le président, font le choix de présenter un projet de loi global. Le volet sur l'interdiction des armes d'assaut a notamment été rajouté parce qu'il n'avait que peu de chance de remporter l'adhésion du Sénat. Le contenu du VCCLEA est si vaste qu'il se heurte d'une part à l'opposition de la droite et des démocrates les plus conservateurs car ils refusent les mesures légiférant davantage l'usage des armes à feu, et d'autre part au refus de l'aile gauche démocrate qui désapprouve la proposition d'extension de la peine capitale contenue dans le volet répressif du VCLEA. Clinton reçoit en ce sens des mises en garde du caucus noir ${ }^{40}$ de la Chambre basse qui craint que les préjugés raciaux ne pèsent encore davantage dans l'application de la peine de mort.

Bill Clinton décide de passer en force. Il part plus franchement à la chasse aux votes, en jouant sur les deux tableaux. Il promet aux républicains de réduire les dépenses liées aux programmes de prévention à hauteur de 3,2 milliards de dollars tout en mettant en avant ces mêmes programmes face aux démocrates. Les démocrates les plus modérés mettent en garde le président contre les effets collatéraux du volet sur les armes. Ils demandent au président de le retirer sous peine de les affaiblir. Clinton refuse et c'est un projet de loi légèrement modifié qui est finalement approuvé par les deux Chambres. La clause tant controversée interdisant les armes d'assaut demeure inchangée, condamnant de ce fait de nombreux démocrates modérés du Sud qui perdront leurs sièges. Seules les dépenses liées à la prévention sont revues à la baisse. Avec 1,8 milliards de dollars, le budget alloué au VCCLEA en fait la loi anti criminalité la plus ambitieuse depuis la loi Safer Streets de 1968.

La signature du VCCLEA attire de nombreuses critiques. La droite s'empare du dossier à l'approche des élections parlementaires. La lutte contre la délinquance est l'un des nombreux sujets de campagne qui serviront à la fois à cimenter une victoire historique du 
parti républicain au Congrès et à asseoir la mainmise des conservateurs sur le Parti républicain. Les chefs de file républicains des deux Chambres intègrent la question sécuritaire dans leurs programmes de campagne. Dans son Contrat pour l'Amérique, le député Newt Gingrich de Georgie propose un plan intitulé The Taking Back Our Streets Act dont la terminologie fait écho aux accents populistes du discours de Pat Buchanan de 1992. Gingrich y réclame l'allongement des peines carcérales, davantage de bâtiments pénitentiaires, le durcissement des conditions de détention et la réduction des dépenses prévues par le VCCLA. Les sénateurs républicains se montrent quant à eux plus prudents. Contrairement au Contrat pour l'Amérique qui préconise la mise en application des mesures au cours des cent premiers jours du Congrès à venir, le plan Seven More in '94 du chef de file républicain le sénateur Bob Dole se veut plus vague. La formule qui avait été trouvée par l'éditorialiste conservateur William Safire (1929-2009) fait référence aux sept sièges sénatoriaux manquants au Parti républicain pour qu'il redevienne majoritaire. Rompu aux pratiques parlementaires, Dole connaît les écueils inhérents au processus législatif et mesure le poids du veto présidentiel.

\section{Les années 2010 : une tendance à l'apaisement}

Par le biais de l'étude de cas du VCCLEA, nous avons démontré dans cet article que la lutte contre la délinquance a représenté un enjeu dans la guerre culturelle et électorale entre conservateurs et progressistes. En d'autres termes, le thème de la lutte contre la délinquance a nourri à la fois l'ascension de l'aile conservatrice au sein du Parti républicain et la guerre culturelle qui a culminé dans les années 1990. Nous souhaitons maintenant aborder le VCCLEA dans sa relecture et voir ce que la réévaluation de ce texte de loi majeur peut nous dire à propos de l'état actuel du traitement de la question sécuritaire aux États-Unis.

Dans un contexte plus apaisé que celui des débats présidant à sa signature, nous entendons analyser l'évolution de la perception de l'efficacité du VCCLEA. Il nous semble pertinent de parler de deux vagues distinctes dans l'évaluation du VCCLEA. La première concerne les avis prononcés à l'issue de la présidence Clinton. D’une part, les défenseurs du VCCLEA ont très tôt fait la corrélation entre la baisse nationale de la criminalité et la mise en application du VCCLEA. Les chiffres du Bureau of Justice semblent corroborer cette thèse. Le taux national de criminalité a décru à partir de 1993. De 1993 à 1998, les crimes violents ont diminué de $21 \%$. En 1997, la moitié des crimes violents ont été commis par une personne connue de la victime, ce qui confirme le bien-fondé de la lutte de Biden pour un meilleur traitement juridique des violences faites aux femmes. Il convient toutefois de nuancer la corrélation qui a été établie entre VCCLEA et la baisse de la criminalité car la période de mise en application du VCCLEA correspond à une baisse du nombre de consommateurs de crack et au vieillissement de la génération d'après-guerre ( baby boomers). D'autre part, les détracteurs du VCCLEA contestent l'efficacité du déploiement massif des forces de l'ordre. La Heritage Foundation, think tank conservateur de renom, reprend dès 2002 les résultats d'une étude qui affirme que le chiffre de 100000 policiers est erroné et avance à la place celui de $68000^{41}$. On peut ajouter à cette critique partisane celle plus indépendante du Government Accountability Office $e^{42}$ selon laquelle l'augmentation des effectifs de police n'aurait réduit le taux de criminalité global que de $1,8 \%$ et de 2,5\% pour les crimes violents au cours de la période 1993-2000 (2005). 
seconde vague d'évaluations du VCCLEA est apparue dans les années 2010 après l'élection de Barack Obama. En 2008, le candidat Obama avait reconnu sans réserve l'efficacité du VCCLEA pour justifier le choix de son co-listier Joe Biden :

Il y a quinze ans de cela, les communautés américaines étaient trop nombreuses à être victimes de la violence et de l'insécurité. Joe Biden a donc amené les républicains et les démocrates à travailler ensemble et à voter pour la loi contre le crime de 1994, ce qui a permis l'envoi de 100000 policiers dans les rues et qui a amorcé une chute du taux de criminalité de huit ans dans tout le pays ${ }^{43}$.

Le choix de Biden pour occuper la vice-présidence était en grande partie guidé par le besoin d'Obama de combler son manque d'expérience en politique étrangère et de rallier les démocrates les plus modérés. Pourtant, les choses changent une fois que l'administration Obama est en place. Les critiques à l'encontre du VCCLEA se multiplient. La forte hausse du taux national d'incarcération est pointée du doigt par la communauté scientifique et par le monde politique. En avril 2014, un rapport de la National Academy of Sciences indique que le niveau d'incarcération atteint par les États-Unis ne peut plus être justifié par les bénéfices sociaux qui peuvent en résulter. La question sécuritaire revient sur le devant de la scène médiatique après chaque fusillade comme la récente tuerie de Charleston du 17 juin 2015. Bien que le jeune inculpé Dylann Roof ait été arrêté peu de temps auparavant pour détention de stupéfiant, il est passé entre les mailles du filet et a pu acquérir une arme.

Aujourd'hui, l'heure n'est plus à la surenchère comme du temps où la guerre culturelle faisait rage. Désormais, droite et gauche dénoncent les effets pervers du VCCLEA et notamment la création d'un système carcéral sans précédent. En 2010, le chiffre record de 2,2 ?millions de détenus est atteint ${ }^{44}$. La population carcérale du pays représente $20 \%$ de la population carcérale mondiale alors que les Américains représentent $5 \%$ de la population mondiale. La population noire est la plus touchée puisqu'on estime qu'un Noir né aujourd'hui a une chance sur trois de se retrouver derrière les barreaux au moins une fois dans sa vie alors que cette probabilité tombe à une chance sur 6 pour un membre de la minorité hispanique et à une sur 17 s'il est de race blanche ${ }^{45}$. Dans son livre coup de poing de 2014, l'activiste de gauche Michelle Alexander compare le système carcéral américain aux lois ségrégationnistes de Jim Crow. Elle affirme que tous deux ont eu pour effet de créer et perpétuer une " hiérarchie raciale $»^{46}$. La culture populaire n'est pas en reste puisque l'émission humoristique Last Week Tonight with John Oliver du 20 juillet 2014 dénonçait aussi l'incarcération de masse. Par le biais de ses marionnettes, John oliver mettait le doigt sur le nombre record de deux millions de prisonniers ainsi que sur le caractère « un peu draconien, et très raciste $\|^{47}$ des lois anti-drogue qui était à l'origine de l'incarcération d'un très grand nombre d'Afro-américains et de populations hispaniques.

41 Notons que l'accroissement de la sévérité pénale américaine a posé question et nourri le débat scientifique. De nombreux spécialistes américains et européens en sociologie du crime se sont penchés sur la spécificité du système répressif américain ${ }^{48}$. La tradition d'égalitarisme liée à une histoire nationale dépourvue d'époque féodale, le poids de la religion, celui de l'esclavage et le rejet de l'intervention étendue de l'État sont autant de facteurs qui ont été avancés. Le virage néolibéral qu'a pris la société américaine a également été invoqué pour établir une corrélation entre l'économie néolibérale et l'insécurité sociale ressentie. Quant au mouvement conservateur, il n'est pas en reste puisque la nouvelle pénologie considère que l'implication des élus conservateurs a intensifié la demande de sanctions par la population. Si le constat d'échec de 
l'incarcération massive mène les membres des blocs progressistes et conservateurs à réfléchir à des solutions nouvelles, il n'en demeure pas moins que le débat sur des sujets sensibles comme la législation des armes à feu est toujours aussi passionné. Les sondages menés par le très sérieux Pew Research depuis 1994, c'est-à-dire l'année du VCCLEA, montrent que la proportion d'Américains ayant des idées conservatives ou progressistes a doublé en vingt ans, passant de $10 \%$ à $21 \%$. En conséquence, un partisan du Parti républicain a beaucoup plus de chance de présenter des idées conservatrices qu'avant et l'inverse est vrai pour un partisan du Parti démocrate ${ }^{49}$.

42 Si l'existence d'une réflexion commune réunissant progressistes et conservateurs pour réformer le système judiciaire et pénitentiaire ne semble pas forcément évidente, le caractère nécessaire et bipartisan de cette réflexion est admis par de nombreux acteurs politiques. De nombreuses initiatives bipartisanes au niveau des États ont vu le jour notamment pour lutter contre le trafic de drogue et l'explosion des incarcérations. Au niveau national, la tentative la plus tangible s'est concrétisée avec la récente sortie d'un livret du Brennan Center (un think tank progressiste spécialisé dans la lutte contre le crime) portant sur les solutions à apporter à un système judiciaire et carcéral défaillant ${ }^{50}$. On y retrouve les interventions de neuf des candidats à la présidence de 2016 avec à gauche Hillary Clinton, Martin O'Malley et Jim Webb, et à droite Chris Christie, Ted Cruz, Scott Walker, Rand Paul, Rick Perry, Marco Rubio ${ }^{51}$. Plus inattendue est la tentative de conciliation bipartisane qui unit les forces des frères Koch, multimillionnaires et libertariens, et du Center for American Progress, think tank progressiste. Depuis février 2015, tous deux mènent une campagne de réforme pénale commune intitulée The Coalition for Public Safety.

En somme, la question sécuritaire est posée en de nouveaux termes: comment faire baisser le taux de délinquance sans recourir systématiquement à l'incarcération? Dans cet effort bipartisan, conservateurs et progressistes conservent chacun leur prisme propre. Les libertariens (libertarians) mettent en avant la dignité humaine et demeurent fidèles à leur demande de moins d'interventionnisme étatique. Les conservateurs fiscaux estiment que le coût du système carcéral est trop élevé tandis que les conservateurs sociaux sont plus sensibles à l'argument de la rédemption. Les démocrates quant à eux mettent en avant la cohésion nécessaire à la bonne marche de la société. Hillary Clinton évoque à ce propos les émeutes raciales de Ferguson de 2014 consécutives la décision de jurys populaires de ne pas poursuivre des policiers blancs pour le meurtre des jeunes Noirs Michael Brown à Ferguson et Eric Garner à New York.

Il nous semble pertinent de s'attarder sur le début de mea culpa de Bill Clinton ainsi que sur le renouveau réformateur du côté conservateur. Au cours de l'année 2015, Bill clinton a fait plusieurs fois état de ses regrets quant à la politique d'incarcération massive qui sévit suite au VCCLEA. Il s'adressait le 7 mai à une journaliste de CNN et le 15 juillet à la National Association of the Advancement of Colored People, l'organisation historique des droits civiques. Certes, on ne peut s'empêcher de penser que les propos de Bill Clinton s'inscrivent dans le prolongement de la campagne présidentielle de son épouse Hillary. Celle-ci a fait de la réforme du système carcéral l'une de ses promesses de campagne. Néanmoins, la reconnaissance même timide des effets pervers du VCCLEA est actée. Dans la préface du livret du Brennan Center, Bill Clinton revient sur ses choix en matière de politique sécuritaire. Il explique que les fortes attentes des Américains l'avaient amené à signer un texte de loi tel que le VCCLEA. Les Américains étaient en effet $37 \%$ en 1994 à placer la lutte contre la criminalité en tête de leurs préoccupations: "Nous avons agi 
pour apporter une réponse à une crise nationale réelle. Mais beaucoup de choses ont changé depuis. Il est temps de porter un regard neuf ce sur ce qui a fonctionné, ce qui n'a pas fonctionné, et ce qui a produit des conséquences non intentionnelles et durables. Beaucoup de nos lois ont bien fonctionné, surtout celles qui ont mis davantage de policiers dans les rues. Mais de nombreuses lois étaient trop étendues au lieu d'être taillées de manière appropriée $»^{52}$. Ce que Clinton passe sous silence, ce sont les pressions de la guerre culturelle qu'il subissait. Malgré le caractère vague de son discours (au demeurant compréhensible au vu du rôle prépondérant qu'il a joué dans le vote du VCCLEA), Bill Clinton reconnaît que la trop grande étendue du VCCLEA a permis certaines dérives.

À droite, la réforme en matière de lutte contre la criminalité a aussi pris le visage de Right on Crime. Il s'agit d'une initiative lancée par l'avocat conservateur Marc Levin en 2010 et qui est abritée par le think tank conservateur Texas Public Policy Foundation. Right on Crime s'appuie sur une stratégie du consensus par l'adhésion à des idées communes et non à une proposition de loi spécifique. Right on Crime entend convaincre les dirigeants conservateurs de souscrire aux principes suivants: la transparence, la liberté individuelle, le rapport entre coût et efficacité, la réhabilitation des détenus, l'intervention limitée de l'État, le soutien aux victimes, et une préférence pour les solutions où interviennent la famille et la communautés3. Marc Levin fait d'ailleurs partie des contributeurs du livret du Brennan Center. Enfin, notons que Newt Gingrich, l'architecte de la révolution républicaine de 1994 à la Chambre des représentants compte parmi les soutiens de Right on Crime.

\section{Conclusion}

Le VCCLEA demeure une étape incontournable pour comprendre l'évolution du traitement de la délinquance aux États-Unis. Il doit sa naissance à une société marquée par un virage néolibéral et la « culture du contrôle ». Les conservateurs américains y sont porteurs d'une pénologie de la vengeance et du bon droit. Leur action en ce sens a progressivement cimenté la construction d'une société néolibérale du risque. La dureté de la bataille législative pour le passage du VCCLEA a notamment exposé à la nation la profondeur du clivage entre les cadres de pensée progressiste et conservateur autour de la question sécuritaire.

La relecture du VCCLEA permet d'identifier une nouvelle et troisième phase du traitement de la question sécuritaire dans la société américaine. Si cette nouvelle phase est marquée par un début de réflexion bipartisane sur la question sécuritaire, et c'est là son trait distinctif, elle reste toutefois l'héritière de la phase précédente, autrement dit de la culture du contrôle. Les tensions partisanes sur la question de la lutte contre la délinquance demeurent même si la bataille pour l'appartenance politique ne semble plus être d'actualité. On voit mal en effet comment les conservateurs pourraient accepter des propositions progressistes telles que la dépénalisation des drogues ou la régularisation des armes. De même, les progressistes ne sauraient accepter des solutions qui agréent les conservateurs comme par exemple la privatisation du système carcéral. De plus, des sujets continuent d'agiter les partis en interne comme la peine de mort ou encore la restauration de droit de vote des anciens détenus. Enfin, les récents développements de la vie politique américaine posent la question des répercussions éventuelles de la campagne de Donald Trump sur l'effort de réflexion bipartisan actuel. 


\section{BIBLIOGRAPHY}

Bibliographie

Alexander, M., The New Jim Crow : Mass Incarceration in the Age of Color Blindness, New York, New Press, 2010.

Baer, K., Reinventing Democrats : the Politics of Liberalism from Reagan to Clinton, Lawrence, University Press of Kansas, 2000.

Bélanger, E. \& Bonnie M., 'Issue Salience, Issue Ownership and Issue-based Vote Choice', Electoral Studies, 2008, 3, 27, p. 477-491.

Biden, J., Promises to Keep : On Life and Politics, New York, Random House Paperback, 2008.

Body-Gendrot, S., 'La politisation du thème de la criminalité aux États-Unis', Déviance et Société, 1999, 23, 1, p. 75-99.

Budge I. \& Farlie, D., Explaining and Predicting Elections : Issue Effects and Party Strategies in twentythree Democracies, London, Allen and Unwin, 1983.

Chettiar, I. \& Waldman, M., (Eds.), Solutions : American Leaders Speak Out on Criminal Justice, Washington, Brennan Center for Justice, 2015.

Clinton, B. \& Gore, A., Mandate for Change, Little Rock, First edition, 1992.

Doherty, C., '7 Things to Know about Polarization in America', Pew Research Center, June 12, 2014.

Etzioni, A., New Communitarian Thinking : Persons, Virtues, Institutions and Communities, Charlottesville, University of Vieginia Press, 1995.

Ewing, W., Martinez, D. \& Rumbaut, R., 'The Criminalization of Immigration in the United States', American Immigration Council, July 2015.

Fiorina, M., Abrams, S. \& Pope, J., Culture War? The Myth of a Polarized America, New York, Pearson Longman, 2004.

From, A., The New Democrats and the Return to Power, St Martin's Press, 2013.

Garland, D., The Culture of Control: Crime and Social Order in Contemporary Society, Chicago, University of Chicago Press, 2001.

Garland, D., 'Adaptations politiques et culturelles des sociétés à forte criminalité', Déviance et Société, 2007, 31, 4, p. 387-403.

Harcourt, B., The Illusion of Free Markets : Punishment and the Myth of Natural Order, Cambridge MA, Harvard University Press, 2011.

Holian, D., 'He’s Stealing my Issues : Clinton's Crime Rhetoric and the Dynamics of Issue Ownership', Political Behavior, 2004, 26, 2, p. 95-124.

Kappeler, V. \& Gaines, L., Community Policing : A Contemporary Perspective, Cincinatti, Lexis Nexis/ Anderson Publishing, (sixth edition), 2012.

Keller, ?B., 'Prison Revolt', The New Yorker, June 29th, 2015.

Kelling, G. \& Wilson J., Broken Windows, The Atlantic Monthly, March, 1982. 
Lacey, N., The Prisoner's dilemma : Political Economy and Punishment in Contemporary Democracies, New York, Oxford University Press, 2008.

Perlstein, R., Before the Storm : Barry Goldwater and the Unmaking of the American Consensus, New York, Hill \& Wang, 2001.

Petrocik, J., 'Issue Ownership in Presidential Elections, with a 1980 Case Study', American Journal of Political Science, 1986, 40, 3, p. 825-850.

Petrocik, J., William, B. \& Hansen, G., 'Issue Ownership and Presidential Campaigning, 1952-2000', Political Science Quaterly, 2003, 118, 4, p. 599-626.

Roesch, L., Les États-Unis : De l'« État-Providence » à l'État pénal, Lormont, Le Bord de l’Eau, 2013.

Saad, L., 'US Liberals at Record 24 ? \%, but Still Trail Conservatives', Gallup, January 9, 2015, http://www.gallup.com/poll/180452/liberals-record-trail-conservatives.aspx, consulté le 29 novembre 2015.

Shermer, E., Barry Goldwater and the Remaking of the American Political Landscape, Tucson, University of Arizona Press, 2013.

Spector, D., 'Sur une prétendue contradiction de l'idéologie libérale. Forum autour du livre de Bernard Harcourt', La Vie des Idées, 7 juin 2011, http://www.laviedesidees.fr/Sur-une-pretenduecontradiction-de.html, consulté le 26 novembre 2015.

Stokes, D., 'Spatial Models of Party Competition', American Political Science Review, 1963, 57, 2, p. 368-377.

Sasson, T., Crime Talk : How Citizens Construct a Social Problem, Hawthorne, Aldine de Gryuter, 1995.

Shafer B. \& Johnston R., The End of Southern Exceptionalism. Class, Race and Partisan Change in the Postwar South, Boston, Harvard University Press, 2009.

Simon, J. \& Feeley, M., 'The Form and Limits of the New Penology', in Blomberg T. \& Cohen, S., Punishment and Social Control, New York, Aldine De Gruyter, p. 75-116.

Van der Brug, W., 'Issue Ownership and Party Choice', Electoral Studies, 2004, 23, 2, p. 209-233

Walzer, M., Spheres of Justice: A Defense of Pluralism and Equality, New York, Basic Books, 1983.

Whitman, J., Harsh Justice : Criminal Punishment and the Widening Divide between America and Europe, New York, Oxford University Press, 2005.

Witcover J., Joe Biden : A Life of Trial and Redemption, New York, William Morrow, 2010.

Zhao, J., Scheider, M. \& Thurman, Q., 'Funding Community Policy to Reduce Crime', Criminology and Public Policy, 2002, 2, 1, p. 7-32.

\section{NOTES}

1. Les émeutes de Los Angeles de 1992 sont nées de la décision d'un jury majoritairement blanc de ne pas sanctionner des policiers blancs qui avaient arrêté puis battu Rodney King, un homme noir, après un excès de vitesse. La vidéo de la scène avait fait le tour du pays et provoqué la colère des populations noires de la ville.

2. «My friends, this election is about much more than who gets what. It is about who we are. It is about what we believe. It is about what we stand for as Americans. There is a religious war going on in our country for the soul of America. It is a cultural war, as critical to the kind of nation we will one day be as was the Cold War itself. (...) Friends, in those wonderful 25 weeks, the saddest 
days were the days of the bloody riot in LA, the worst in our history. But even out of that awful tragedy can come a message of hope. (...) Here were 19-year-old boys ready to lay down their lives to stop a mob from molesting old people they did not even know. And as they took back the streets of LA, block by block, so we must take back our cities, and take back our culture, and take back our country ", Buchanan P., Discours à la convention nationale républicaine, 17 août 1992.

3. Le flou conceptuel qui entoure le recours aux termes "progressiste " (tiré du vocable du XIXe siècle) et «liberal » témoigne de la victoire des conservateurs sur les termes du débat politique. Ce point sera approfondi dans la suite de l'article.

4. Simon \& Feeley (2003).

5. Garland (2001 \& 2007).

6. Budge I. \& Farlie, D. ?(1983).

7. «the ability to resolve a problem of concern to voters. It is a reputation for policy and program interests, produced by a history of attention, initiative, and innovation toward these problems », Petrocik (1984, p. 826).

8. Petrocik (1996) ; Petrocik, Benoît \& Hansen (2003).

9. Stokes (1963).

10. Van der Brug (2004); Bélanger \& Meguid (2008).

11. Pour dissiper tout malentendu, nous précisons que nous utilisons le terme « idéologie » et son adjectif «idéologique " dans une acception non marxiste (laquelle suppose l'existence d'un système théorique plus ou moins construit et dont la visée principale est le maintien d'une oppression de classe). Nous préférons nous limiter à un sens plus courant et moins critique : l'ensemble des idées qui structurent les professions de foi publiques et les actions proposées par un parti politique.

12. Body-Gendrot (1999).

13. «Tonight there is violence in our streets, corruption in our highest offices, aimlessness among our youth, anxiety among our elders (...)».

14. Perlstein (2001); Shermer (2013).

15. On peut définir le «one Solid South » comme le regroupement d'électeurs blancs du Sud (anciennement confédéré) uniformément conservateur, raciste, opposé aux syndicats et qui a voté massivement pour le Parti démocrate de la fin de la guerre de Sécession (1861-1865) jusqu'au début des années 1970.

16. Black (1996); Shafer \& Johnston (2009).

17. Depuis le recensement de 1940 , le Sud est la région ayant la population la plus importante aux États-Unis. Le poids du Sud ne cesse de s'accentuer puisque le dernier recensement indique que le Sud détient le taux d'accroissement de population le plus important de la nation (soit 14,3\%) de 2000 à 2010.

18. Ils pratiquent notamment le gerrymandering. Lors du redécoupage électoral décennal, les élus conservateurs cherchent à augmenter la compétitivité de leurs candidats en regroupant l'électorat fortement démocrate dans certains comtés.

19. Saad (2015).

20. «We must reject the idea that every time a law's broken, society is guilty rather han the lawbreaker. It is time to restore the American precept that each individual is accountable for his actions ».

21. Nancy Reagan se servit de son statut de Première dame pour promouvoir une campagne de sensibilisation à la drogue auprès des enfants et des jeunes adultes dès 1982. Elle se rendit dans de nombreux centres de désintoxication pour les jeunes et apparut à la télévision seule (dans des programmes de divertissement) ou avec son époux (pour des allocutions plus formelles) pour promouvoir la campagne de lutte contre la drogue "Just say no ». Son message de prévention laissa la place à un plaidoyer en faveur de la lutte contre le trafic international des stupéfiants lorsqu'elle se présenta devant l'Assemblée générale des Nations Unies en octobre 1988. 
22. Roesch (2013, p. 96).

23. Harcourt, 2011 ; Spector (2011).

24. Body-Gendrot (1999, p. 77).

25. Massachusetts Executive Office of Public Safety and Security, 'Violent Crime in Massachussetts : a 25-Year Retrospective', Annual Policy Brief (1988-2012), February 2014.

26. Traduction proposée: «Des laisser-passer du week-end pour les détenus: la position de Dukakis ».

27. Witcover (2010).

28. Le nom et adjectif community ne peut se traduire par le terme français communautaire car il recouvre un sens particulier et différent du vocable français. Community désigne un groupe de personnes vivant en un même endroit (quartier, ville) et ayant des intérêts communs. En raison parfois du peuplement de certains quartiers, community réfère aussi à l'ethnicité, la race ou la religion.

29. Kappeler \& Gaines (2012).

30. Walzer (1983).

31. «More deeply, we were troubled that many Americans are rather reluctant to accept responsibilities. We were distressed that many Americans are eager to spell out what they are entitled to, but are slow to give something back to the community and to others. We adopted the name 'communitarian' to emphasize that the time had come to attend to our responsibilities to our communities », Etzioni (1995, IV).

32. La NRA a notamment assis son pouvoir en imposant son interprétation du second amendement de la Constitution. Cet amendement à l'origine défend le droit à l'existence d'« une milice bien organisée étant nécessaire à la sécurité d'un État libre, (et) le droit qu'a le peuple de détenir et de porter des armes ne sera pas transgressé ». Selon la NRA, ces droits collectifs sont également individuels et le second amendement garantirait donc le droit d'un individu à détenir une arme.

33. « For too long, a majority of this body has preferred politics to pragmatics. Last Congress, we had the opportunity to pass needed crime legislation that contained program after program supported by democrats and republicans alike. Instead we remained mired in disagreement over a few provisions that sparked political heat out of all proportion to their relative importance to effectively fighting crime ».

34. William Renhquist était l'ancien conseiller juridique de Barry Goldwater pendant la campagne présidentielle de 1964. En 1971, il fut nommé juge à la Cour suprême par le président Nixon.

35. « When I came to Washington, I would read editorials from the prominent newspapers saying that if you cared about the deficit and crime and welfare, you were stealing Republican issues. And I said, now, wait a minute. (...) I think those are American issues the Democratic Party has done very well on, and I don't understand all this ", Clinton, ?B., Remarks at a Democratic Governors' Association Reception, November 12, 1997.

36. David B. Holian (2004).

37. Le Personal Responsibility and Work Opportunity Act de 1996 réforme en profondeur les modalités d'attribution des aides financières et alimentaires à destination des populations défavorisées. Il remplace le programme Aid to Families with Dependent Children par des sommes annuelles fixes données aux États pour le programme Temporary Assistance to Needy Families. La réforme réduit les dépenses fédérales sur les bons alimentaires, le complément de revenu social, les programmes de nutrition pour l'enfance et elle diminue les sommes allouées aux services sociaux. Parallèlement, la réforme de l'aide sociale augmente les fonds alloués à la garde des enfants issus des familles à faibles revenus. Enfin, ce texte de loi impose un critère de citoyenneté américaine pour bénéficier de certains programmes des aides sociales.

38. Baer (2000); From (2014). 
39. La flibuste est une man ?uvre d'obstructionnisme qui consiste à faire reculer l'adoption d'un projet de loi en allongeant la durée des débats parlementaires. Seul un vote de clôture peut contrer la flibuste. Au Sénat, il faut soixante voix pour obtenir le vote de clôture. L'adoption d'un texte de loi nécessite donc non pas cinquante ou cinquante-et-un votes comme indiqué dans la Constitution mais bien soixante.

40. Le caucus noir du Congrès défend les intérêts de la minorité afro-américaine.

41. Zhao, Scheider \& Thurman (2002, p. 7-32).

42. Le Government Accountability Office est une agence indépendante qui travaille pour le Congrès et dont l'objet est de vérifier le bon usage des dépenses fédérales.

43. Barack Obama, Remarks Introducing Senator Joseph Biden as the 2008 Democratic VicePresidential Nominee in Springfield, Illinois, August 23, 2008.

44. U.S. Bureau of Justice Statistics, Correctional Populations in the United States, 2011, 1, 3 tbl.2, Nov. 2012.

45. Report of the Sentencing Project to the United Nations Human Rights Committee : Regarding Racial Disparities in the United States Criminal System, August 2013.

46. Alexander (2014, p. 16).

47. « a little draconian, and a lot racist ».

48. Whitman (2005); Lacey (2008).

49. Doherty (2014).

50. Chettiar \& Waldman (2015).

51. On remarquera l'absence de la liste de Donald Trump, candidat victorieux des primaires républicaines de 2016. L'étendue du populisme de sa campagne en font un candidat controversé au sein même du parti de l'éléphant. Trump cherche à courtiser les citoyens qui ont le sentiment d'être déclassés dans une nation en crise. Confronté à une carte électorale défavorable et à un Parti républicain affaibli, il adopte une stratégie de l'outrance qui vise à amener aux urnes les électeurs blancs habituellement abstentionnistes. S'attaquer à l'immigration illégale pour traiter la question sécuritaire permet de rappeler à tous l'échec de la réforme d'immigration d'Obama.

Son discours d'investiture en est un exemple. Dans un contexte de fortes tensions raciales, il associe la délinquance à l'immigration illégale: «Nous serons un pays de générosité et de chaleur. Mais nous serons aussi un pays où règne l'ordre public. (...) Presque 180000 sans-papiers détenant un casier judiciaire, dont l'expulsion a été ordonnée, circulent librement dans le pays et menacent de paisibles citoyens ». Cet argument va naturellement à l'encontre des études régulièrement menées sur la question lesquelles montrent que les immigrants sont moins enclins que le reste de la population américaine à violer la loi. Un rapport récent de l'American Immigration Council indique que le taux de crimes violents a baissé de 48 ? \% de 1990 à 2013 tandis que le nombre d'immigrants illégaux a triplé dans le même temps (Ewing, Martinez \& Rumbaut, 2015).

52. « We acted to address a genuine national crisis. But much has changed since then. It's time to take a clear-eyed look at what worked, what didn't, and what produced unintended, long-lasting consequences. So many of these laws worked well, especially those that put more police on the streets. But too many laws were overly broad instead of appropriately tailored ", Chettiar \& Waldman (2015, p. 4).

53. Keller (2015). 


\section{ABSTRACTS}

The issue of crime fighting has constituted an integral part of the conservative discourse in the United States since the 1950s. The political right has gradually appropriated this key theme in an attempt to dominate the Republican Party and to throw the Democratic Party off course. This study of the Violent Crime Control and Law Enforcement Act of 1994, the most important anticrime law of the second half of the twentieth century, reveals at the same time the cultural war conducted by the conservative movement as well as its consequences. The analysis of the origins of the VCCLEA, its implementation, and its recent revision allow the author to identify three phases in the approach to security issues in the United States. The most recent phase in the debate on crime, during the presidential elections of 2016, has been characterised by a relative relaxation in the partisan tensions, at least in what concerns the question of incarceration.

La question de la lutte contre la délinquance fait partie intégrante du discours du mouvement conservateur américain depuis son émergence dans les années 1950. Les conservateurs se sont progressivement approprié ce thème porteur afin de conquérir le Parti républicain et de mettre en déroute le Parti démocrate. L'étude du Violent Crime Control and Law Enforcement Act de 1994, la loi anticriminalité américaine la plus importante de la seconde moitié du XXe siècle, illustre à la fois la guerre culturelle menée par les conservateurs et ses dérives. L'analyse des racines et de la mise en oeuvre du VCCLEA couplée à sa relecture récente permet d'identifier trois phases dans le traitement de la question sécuritaire aux États-Unis. Dans le contexte électoral des primaires de 2016, la dernière phase est marquée par un apaisement relatif des tensions partisanes du moins sur la question carcérale.

\section{AUTHOR}

\section{DIANE BÉNÉDIC-MEYER}

Docteur en études anglophones, Diane Bénédic-Meyer est spécialiste de l'histoire politique et socio-économique des États-Unis de la fin du XX ${ }^{\mathrm{e}}$ siècle et du début du XXI ${ }^{\mathrm{e}}$. Sa thèse de doctorat, soutenue en 2014, porte sur la modernisation idéologique du parti démocrate aux États-Unis de 1980 à 2011. Diane Bénédic-Meyer a publié aux États-Unis des articles sur le parti démocrate (Chapman, R.(Ed.), Encyclopedia of the Culture Wars, Issues, Voices, and Viewpoints, Armonk, M.E. Sharpe, 2009). Elle participe régulièrement à la préparation de l'agrégation d'anglais en donnant des communications et en publiant des articles dans des revues spécialisées (Les années Clinton: la personnalisation excessive de l'exécutif in Robert, F.(Dir.), L'empire de l'Exécutif: la Présidence des États-Unis de Franklin Roosevelt à George W. Bush (1933-2006), Ellipses, 2008, p. 163-176. Ses recherches en cours portent sur la notion d'entreprenariat politique dans le but d'identifier des centres de pouvoir autres que les partis politiques. - docto67@hotmail.com 